\title{
Anti-viral therapy is associated with improved survival but is underutilised in patients with hepatitis B virus-related hepatocellular carcinoma: real-world east and west experience
}

\author{
V. L. Chen ${ }^{1,2}$ \\ M.-L. $\mathrm{Yeh}^{3}$ \\ A. K. $\operatorname{Le}^{4}$ \\ M. Jun ${ }^{5}$ \\ W. K. Saeed ${ }^{6}$ \\ J. D. Yang ${ }^{7}$ | \\ C.-F. Huang ${ }^{3}$ \\ H. Y. Lee ${ }^{6}$ \\ P.-C. Tsai ${ }^{3}$ \\ M.-H. Lee ${ }^{8}$ \\ N. Giama ${ }^{7}$ \\ N. G. Kim ${ }^{9}$ \\ P. P. Nguyen ${ }^{4}$ \\ H. Dang ${ }^{4}$ \\ H. A. Ali $^{7}$ \\ N. Zhang ${ }^{7}$ \\ J.-F. Huang ${ }^{3}$ \\ C.-Y. Dai ${ }^{3}$ \\ W.-L. Chuang ${ }^{3}$ | \\ L. R. Roberts ${ }^{7}$ \\ D. W. Jun ${ }^{6}$ \\ Y.-S. Lim 5 \\ M.-L. $\mathrm{Yu}^{3}$
}

M. H. Nguyen ${ }^{4}$

${ }^{1}$ Division of Gastroenterology, University of Michigan Health System, Ann Arbor, MI, USA

${ }^{2}$ Department of Medicine, Stanford University Medical Center, Stanford, CA, USA

${ }^{3}$ Hepatobiliary Division, Department of Internal Medicine, Kaohsiung Medical University Hospital, Kaohsiung Medical University, Kaohsiung, Taiwan

${ }^{4}$ Division of Gastroenterology and Hepatology, Stanford University Medical Center, Stanford, CA, USA

${ }^{5}$ Department of Gastroenterology, Asan Medical Center, University of Ulsan College of Medicine, Seoul, Korea

${ }^{6}$ Division of Gastroenterology, Hanyang University Medical Center, Seoul, Korea

${ }^{7}$ Department of Gastroenterology and Hepatology, Mayo Clinic, Rochester, MN, USA

${ }^{8}$ Institute of Clinical Medicine, National Yang-Ming University, Taipei, Taiwan

${ }^{9}$ Stanford University School of Medicine, Stanford, CA, USA

\section{Correspondence}

Dr. M H Nguyen, Division of Gastroenterology and Hepatology, Stanford University Medical

Center, Palo Alto, CA, USA.

Email: mindiehn@stanford.edu

and

Dr. M-L Yu, Kaohsiung Medical University Hospital, Kaohsiung Medical University,

Kaohsiung, Taiwan.

Email: fish6069@gmail.com

Funding information

Taiwan Ministry of Science and Technology, Grant/Award Number: MOST 105-2628-B010-003 MY4

\section{Summary}

Background: Hepatitis B virus (HBV) is the leading cause of hepatocellular carcinoma (HCC) worldwide. It remains incompletely understood in the real world how anti-viral therapy affects survival after HCC diagnosis.

Methods: This was an international multicentre cohort study of $2518 \mathrm{HBV}$-related HCC cases diagnosed between 2000 and 2015. Cox proportional hazards models were utilised to estimate hazard ratios (HR) with $95 \%(\mathrm{Cl})$ for anti-viral therapy and cirrhosis on patients' risk of death.

Results: Approximately, $48 \%$ of patients received anti-viral therapy at any time, but only $17 \%$ were on therapy at HCC diagnosis (38\% at US centres, $11 \%$ at Asian centres). Anti-viral therapy would have been indicated for $>60 \%$ of the patients not on anti-viral therapy based on American criteria. Patients with cirrhosis had lower 5year survival ( $34 \%$ vs $46 \% ; P<0.001$ ) while patients receiving anti-viral therapy had increased 5 -year survival compared to untreated patients ( $42 \%$ vs $25 \%$ with cirrhosis and $58 \%$ vs $36 \%$ without cirrhosis; $P<0.001$ for both). Similar findings were seen for other patient subgroups by cancer stages and cancer treatment types. Antiviral therapy was associated with a decrease in risk of death, whether started before or after HCC diagnosis (adjusted HR 0.62 and 0.79, respectively; $P<0.001$ ).

Conclusions: Anti-viral therapy improved overall survival in patients with HBVrelated HCC across cancer stages and treatment types but was underutilised at both US and Asia centres. Expanded use of anti-viral therapy in HBV-related HCC and better linkage-to-care for HBV patients are needed. 


\section{INTRODUCTION}

Hepatocellular carcinoma (HCC) is the third leading cause of cancer death worldwide with nearly 600000 deaths in $2008,{ }^{1}$ and hepatitis $B$ virus (HBV) infection is the most common cause of HCC. $^{2}$ Up to $20 \%-40 \%$ of patients with HBV infection may develop HCC in the absence of clinically apparent cirrhosis. ${ }^{3,4}$ The advent of highly potent anti-viral therapy has offered the possibility of greatly decreasing the incidence of liver-related complications, including cirrhosis and $\mathrm{HCC}$, in patients with chronic hepatitis $\mathrm{B}(\mathrm{CHB})^{5,6}$

While anti-viral medications are known to reduce the risk of $\mathrm{HCC}$ in patients with $\mathrm{CHB}{ }^{7}$ it is less well understood how they influence the overall survival of patients with established HCC, with most data only focusing on patients undergoing curative therapy for HCC. ${ }^{8}$ A 2013 randomised controlled trial comparing nucleot(s)ide analogues to placebo in patients with HBV-related HCC treated with partial hepatectomy found that anti-viral therapy decreased the risk of HCC recurrence, HCC-related mortality and overall mortality. ${ }^{9}$ These findings are supported by an earlier meta-analysis of 9 cohorts and a national database study showing that HCC patients receiving curative therapy for HCC and treated with anti-viral agents had decreased overall mortality and recurrence rate. $^{10,11}$ Likewise, anti-viral therapy after radiofrequency ablation is associated with decreased HCC recurrence. ${ }^{12}$ The data on anti-viral therapy in patients treated with palliative therapy, including transarterial chemoembolisation (TACE) and sorafenib are more limited, but in the case of TACE, a randomised controlled trial demonstrated that anti-viral therapy increases survival. ${ }^{13-15}$

Currently, the presence of HCC is not considered an indication for anti-viral medications in international guidelines for the management of $\mathrm{CHB}^{16-18}$ In addition, lifelong anti-viral medications may not be reimbursed by third-party payers in certain areas such as Taiwan if patients have HCC but no cirrhosis. ${ }^{19}$ Thus, the question of how much viral suppression can reduce mortality among HBV-related HCC patients can have important policy implications. Related to this issue is the "cascade of care" for patients with $\mathrm{CHB}$, which describes the reasons for which rates of treatment for $\mathrm{CHB}$ are suboptimal. ${ }^{20}$ Many patients with $\mathrm{CHB}$ have not even been diagnosed, and among those who are diagnosed many have not established care with the medical system. ${ }^{21}$ Even among those with access to appropriate medical care, treatment rates of $\mathrm{CHB}$ are suboptimal for numerous reasons including patient loss to follow-up, financial difficulties and misconceptions about indications for anti-viral therapy. ${ }^{22} \mathrm{~A}$ greater understanding of the use of anti-viral therapy in HBV-related HCC may provide further insight into how $\mathrm{CHB}$ patients interact with the healthcare system.

The goal of this study was to examine the effect of anti-viral therapy on overall survival of patients with HBV-related HCC, taking into account the presence or absence of cirrhosis in a real-world cohort of East and West patients.

\section{2 | PATIENTS AND METHODS}

\subsection{Study design and patient population}

We performed an international multicentre cohort study of HBVrelated $\mathrm{HCC}$ at 5 medical centres. The inclusion criteria were $\mathrm{CHB}$ (defined as positive serum hepatitis $B$ surface antigen, detectable HBV DNA, or on anti-viral therapy for a history of $\mathrm{CHB}$ ) and a new diagnosis of HCC (by pathology or imaging based on 2010 American Association for the Study of Liver Diseases criteria). ${ }^{23}$ Patients with prior HCC or liver transplant were excluded. Patients were screened via an ICD-9 diagnosis query for HCC and included in the cohort if the above criteria were met based on individual chart review.

The Kaohsiung Memorial University Hospital cohort included consecutive 1261 patients diagnosed between 2000 and 2015. The Asan Medical Center cohort included 496 randomly selected patients diagnosed between 2005 and 2015. The Stanford University Medical Center cohort included 453 consecutive patients diagnosed with HCC between 2000 and 2014. The Hanyang University Medical Center cohort included 289 consecutive patients seen in clinic between 2005 and 2015. The Mayo Clinic cohort included 44 patients diagnosed between 2005 and 2011, as previously reported. $^{24}$ This study was approved by the Institutional Review Boards at Stanford University (Stanford, CA, USA) and each of the other participating centres.

Selection bias is decreased by the use of consecutive patients. Study size was not pre-determined and was based on the number of patients diagnosed with $\mathrm{HCC}$ between certain time periods.

\subsection{Definition of cirrhosis and anti-viral treatment}

Laboratory data, imaging findings, and $\mathrm{HCC}$ and cancer treatment modalities were obtained from patients' medical records. Patients were designated as having cirrhosis if they were deemed to have cirrhosis based on hepatology notes, or if there was pathological evidence of fibrosis stage 4, clinical evidence of portal hypertension (otherwise-unexplained splenomegaly or platelet count $<120000 / \mu \mathrm{L}$, ascites, or gastroesophageal varices on imaging), prior hepatic decompensation (hepatic encephalopathy, ascites, variceal gastrointestinal bleeding), or laboratory evidence of decreased synthetic function (total bilirubin $>2.0 \mathrm{mg} / \mathrm{dL}$ or international normalised ratio $>1.2$ without alternative explanation). Anti-viral therapy status was determined by chart review and pharmacy records. Criteria for antiviral therapy were based on American Association for the Study of Liver Disease and Asia-Pacific Association for the Study of the Liver guidelines. ${ }^{16,17}$

\section{3 | Tumour staging and survival outcomes}

Tumour characteristics were determined by triphasic computed tomography or magnetic resonance imaging. Patients were followed from the date of diagnosis with HCC and either death or last follow- 
up date in the medical record. For the Stanford and Mayo cohorts, patients not known to be deceased and whose last visit to the medical centre was before January 1 2015, we also performed a National Death Index registry search from 1979-2014. The National Death Index registry is a centralised database of death record information on file in state vital statistics offices with over $90 \%$ completion for most states and $99 \%$ for the state of California where the Stanford cohort is located. ${ }^{25}$ For the Kaohsiung cohort, telephone interview with families was also conducted to obtain additional follow-up data.

\subsection{Anti-viral therapy indications}

Four different standards of anti-viral therapy were used: American Association for the Study of Liver Disease guidelines, ${ }^{16}$ Asia-Pacific Association for the Study of the Liver, ${ }^{26}$ Ministry of Health and Welfare for the Republic of Korea ${ }^{27}$ and National Health Insurance Administration for Taiwan. ${ }^{28}$ Local guidelines were defined as American Association for the Study of Liver Disease guidelines for United States centres, Ministry of Health and Welfare reimbursement criteria for Korean centres and National Health Insurance Administration for the Taiwan centre (Table S1).

\subsection{Statistical analysis}

Descriptive statistics were reported as proportion (\%) for categorical variables, and mean \pm standard deviation (SD) or median (and range) for continuous variables. Normally distributed continuous variables were compared by Student's $t$ tests. Nonparametric statistics were applied when continuous variables were not normally distributed. Chi-squared tests were used to compare categorical variables. In this study, the primary outcome was overall survival of HCC patients. Person-years of follow-up were calculated for each patient as the time from dates of HCC diagnosis to the date of death or to the last date when patients were last known to be alive. Mortality rates by various disease status were calculated and expressed per 100 person-years. Kaplan-Meier methods were utilised to depict the overall survival of patients with or without anti-viral therapy; patients lost to follow-up were censored. Statistical differences in overall survival by various subgroups were compared and examined by log-rank tests. Cox proportional hazards models were used to estimate the hazard ratios (HRs) and $95 \%$ (Cls) relating anti-viral therapy and other risk factors with overall survival. Lead time analysis was performed as previously described. ${ }^{29,30}$ Statistical significance was defined as a two-tailed $P$ value $<0.05$. Missing data were excluded from analysis. All statistical analyses were performed using STATA 11.0 (Stata Corporation, College Station, TX, USA).

\section{3 | RESULTS}

\section{1 | Baseline clinical/tumour characteristics}

The overall cohort consisted of 2518 patients, of whom $73 \%$ had cirrhosis, $81 \%$ were male, and $98 \%$ were Asian. Baseline clinical and laboratory characteristics of the patients in this cohort by anti-viral therapy status are shown in Table 1. Among the patients with cirrhosis, $30 \%$ had had a history of hepatic decompensation with variceal bleeding, hepatic encephalopathy, or ascites, and $38 \%$ had Child-Pugh-Turcotte (CPT) class B or C disease.

In total, $49 \%$ of patients had ever received anti-viral therapy at any time (Figure 1). However, only $17 \%$ were on treatment at time of HCC diagnosis (Figure 1). Proportion of patients receiving anti-viral therapy before their diagnosis of HCC did not differ between those with or without cirrhosis (17.9\% vs $15.3 \%$; $P=0.12$; Figure $\mathrm{S} 1 \mathrm{~A}$ )

TABLE 1 Clinical and tumour characteristics, stratified by antiviral therapy use

\begin{tabular}{|c|c|c|c|}
\hline Characteristic & $\begin{array}{l}\text { No anti-virals } \\
(\mathrm{N}=1283)\end{array}$ & $\begin{array}{l}\text { Anti-virals } \\
(\mathrm{N}=1235)\end{array}$ & $\begin{array}{l}P \\
\text { value }\end{array}$ \\
\hline Age at cancer diagnosis & $59.0 \pm 12.0$ & $56.4 \pm 10.8$ & $<0.001$ \\
\hline Male & $80.0 \%$ & $82.8 \%$ & 0.073 \\
\hline Asian & $98.7 \%$ & $97.9 \%$ & 0.085 \\
\hline Any decompensation & $26.6 \%$ & $20.7 \%$ & 0.001 \\
\hline $\begin{array}{l}\text { Anti-virals before cancer } \\
\text { diagnosis }\end{array}$ & N/A & $35.4 \%$ & N/A \\
\hline \multicolumn{4}{|l|}{ Anti-virals used } \\
\hline Lamivudine & N/A & $37.7 \%$ & $\mathrm{~N} / \mathrm{A}$ \\
\hline Adefovir & & $11.0 \%$ & \\
\hline Telbivudine & & $3.0 \%$ & \\
\hline Entecavir & & $49.0 \%$ & \\
\hline Tenofovir & & $14.3 \%$ & \\
\hline $\begin{array}{l}\text { Interferon (including } \\
\text { pegylated) }\end{array}$ & & $1.9 \%$ & \\
\hline Other & & $0.7 \%$ & \\
\hline Mean Child-Pugh score & $6.6 \pm 1.8$ & $6.4 \pm 1.6$ & 0.012 \\
\hline Liver cirrhosis & $70.0 \%$ & $76.0 \%$ & 0.001 \\
\hline \multicolumn{4}{|l|}{ Child-Pugh class } \\
\hline A & $64.8 \%$ & $67.6 \%$ & 0.008 \\
\hline B & $27.8 \%$ & $28.0 \%$ & \\
\hline $\mathrm{C}$ & $7.4 \%$ & $4.4 \%$ & \\
\hline $\begin{array}{l}\text { Model for end-stage liver } \\
\text { disease score }\end{array}$ & $11.1 \pm 5.2$ & $10.2 \pm 4.1$ & $<0.001$ \\
\hline Maximum tumour size & $6.0 \pm 4.5$ & $4.8 \pm 4.0$ & $<0.001$ \\
\hline \multicolumn{4}{|l|}{ Number of tumours } \\
\hline Unifocal & $44.8 \%$ & $52.1 \%$ & $<0.001$ \\
\hline Multifocal & $55.2 \%$ & $47.9 \%$ & \\
\hline Vascular invasion & $28.5 \%$ & $20.8 \%$ & $<0.001$ \\
\hline Extrahepatic metastasis & $12.8 \%$ & $7.5 \%$ & $<0.001$ \\
\hline \multicolumn{4}{|c|}{ Barcelona clinic liver cancer stage } \\
\hline 0 & $7.3 \%$ & $10.4 \%$ & $<0.001$ \\
\hline A & $24.7 \%$ & $37.3 \%$ & \\
\hline B & $25.8 \%$ & $24.8 \%$ & \\
\hline C & $34.3 \%$ & $22.6 \%$ & \\
\hline D & $8.0 \%$ & $4.9 \%$ & \\
\hline
\end{tabular}




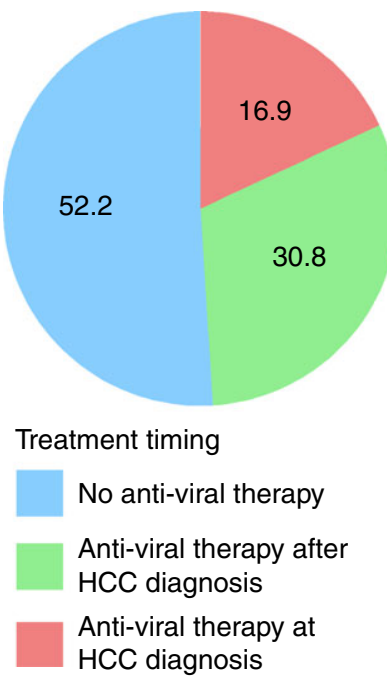

FIGURE 1 Treatment with anti-viral therapy. Percentage of patients receiving treatment with anti-viral therapy, either before hepatocellular carcinoma diagnosis (red), after hepatocellular carcinoma diagnosis (green), or never (blue). Numbers represent percentages of patients in each category

We next focused on the $83 \%$ of patients who were not on anti-viral therapy at time of HCC diagnosis, in particular on whether treatment would have been indicated for these patients. There was considerable variation in whether or not anti-viral therapy was indicated depending on the specific guideline and on cirrhosis status. Over $80 \%$ of patients with decompensated cirrhosis met criteria based on national and international guidelines, while $<10 \%$ of patients without cirrhosis did (Figure 2). The widest variation between guidelines was seen in patients with compensated cirrhosis, where $84 \%$ of patients met American standards while only $4 \%$ met Taiwan reimbursement criteria and $44 \%$ met Korea reimbursement and Asia-Pacific criteria for antiviral therapy $(P<0.0001)$ (Figure 2$)$. Centres in different countries could have patients with different viral characteristics, but even when matching the patients of each country to the reimbursement guidelines in those countries, the overall trend persisted, where anti-viral therapy was more frequently indicated in patients with decompensated cirrhosis than compensated cirrhosis than no cirrhosis (Figure 3).

Of the patients who were not on therapy at time of HCC diagnosis, $37 \%$ were later started on anti-viral therapy (Figure 1). Patients with cirrhosis were more likely to receive anti-viral therapy after HCC diagnosis and more likely to receive anti-viral therapy at any time than those without cirrhosis $(49.9 \%$ vs $43.1 \%$; $P<0.001$; Figure S1A). Of note, anti-viral therapy is reimbursed in Korea for patients with detectable HBV DNA and $\mathrm{HCC}^{27}$ but HBV DNA was detected in $68 \%$ of patients who never received anti-viral therapy after HCC diagnosis.

Though rates of anti-viral therapy were well below $50 \%$ at time of HCC diagnosis at all centres, it was higher at the US centres than the Taiwan or Korea centres (40\% vs $12 \%$ vs $10 \%$; $P<0.001$ for three-way comparison; Figure S1B). After HCC diagnosis, $68 \%$ of the US and $61 \%$ of the Korea centre patients were on anti-viral therapy, compared to only $31 \%$ in the Taiwan centre (Figure S1B; $P<0.001$ for three-way comparison). There was no difference in anti-viral therapy use based on date of HCC diagnosis 2005 or before, 20062010 , or 2011 or later: $52 \%, 52 \%$, and $49 \%$ of patients received anti-viral therapy at any time, respectively, in these time periods $(P=0.22)$.

Patients receiving anti-viral therapy were younger (56.4 vs 59.0 years; $P<0.001)$ and more often had cirrhosis (76\% vs $70 \%$; $P<0.001)$ than those not receiving anti-viral therapy (Table 1). The anti-viral medications most commonly used were entecavir (49.5\%), lamivudine (37.9\%), tenofovir (14.9\%) and adefovir (11.0\%), with little use of telbivudine (3.1\%) and interferon-containing regimens (1.8\%). Patients with cirrhosis more frequently received anti-viral therapy, and those receiving anti-viral therapy were less likely to have decompensated cirrhosis and had lower CPT class and model for end-stage liver disease (MELD) scores than those who did not receive anti-viral therapy $(P<0.1)$. In addition, regardless of cirrhosis status, patients receiving anti-viral therapy had earlier cancer stage, based on Barcelona clinic liver cancer (BCLC) stage, smaller maximum tumour size, and decreased prevalence of multifocal tumours, vascular invasion and extrahepatic metastasis $(P<0.001)$.

Regarding HCC by cirrhosis status, patients with cirrhosis had smaller maximum tumour size than those without cirrhosis (5.2 vs $5.8 \mathrm{~cm} ; P=0.004$ ) but more often had multifocal tumours (55\% vs 42\%; $P<0.001)$. Compared to patients without cirrhosis, HCC patients with cirrhosis also had a greater prevalence of vascular invasion and extrahepatic metastasis, as well as a higher BCLC stage ( $P<0.001$ for all comparisons).

\subsection{Cancer treatment}

Table S2 illustrates cancer treatment based on presence or absence of cirrhosis. Patients with cirrhosis were less likely to receive cancer treatment than patients without cirrhosis $(82.4 \%$ vs $88.7 \%$; $P<0.001)$, particularly partial hepatectomy $(16.4 \%$ vs $39.8 \%$; $P<0.001)$, though they were more likely to undergo $L T(4.4 \%$ vs $1.0 \%$; $P<0.001)$. While patients with cirrhosis more often received liver-directed therapy $(65.5 \%$ vs $53.5 \% ; P<0.001)$, this difference was largely driven by transarterial chemoembolisation rather than curative ablations.

When comparing cancer-directed treatment modalities based on anti-viral therapy status (Table S3), patients receiving anti-viral therapy were more likely to receive any treatment and most individual treatments including resection, LT, and liver-directed therapy. As with cirrhosis, the difference in liver-directed therapy was driven primarily by palliative transarterial chemoembolisation and external radiation therapy.

\section{3 | Mortality rates and overall survival}

Table 2 shows the mortality rates by various disease status and treatment types. In total, there were 1415 deaths after 6384 person-years of follow-up, yielding overall mortality of 22.2 per 100 
Patients not on anti-viral therapy at HCC diagnosis

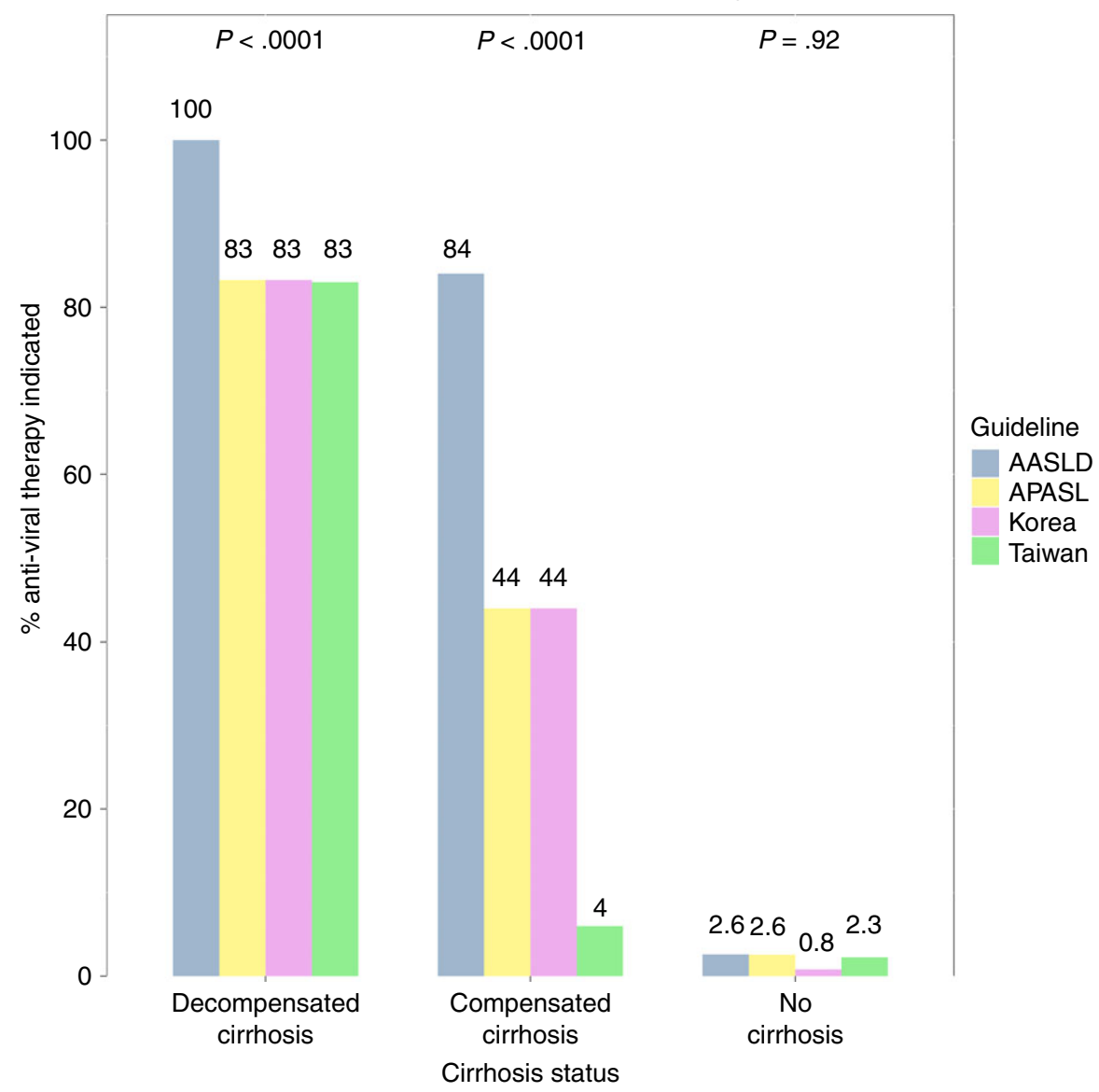

FIGURE 2 Indication for treatment with anti-viral therapy. For patients who were not on anti-viral therapy at time of $\mathrm{HCC}$ diagnosis, y axis shows percentage of patients for whom anti-viral therapy would have been indicated, based on guidelines applied uniformly to all centres. Data are divided based on cirrhosis status: decompensated cirrhosis, compensated cirrhosis and no cirrhosis. Four sets of guidelines were used: AASLD (American Association for the Study of Liver Diseases; grey), ${ }^{16}$ APASL (Asia-Pacific Association for the Study of the Liver; yellow), ${ }^{26}$ Ministry of Health and Welfare for the Republic of Korea (purple) ${ }^{27}$ and National Health Insurance Administration for Taiwan (green) ${ }^{28}$ person-years in the study population. Overall, patients with cirrhosis had increased mortality rate compared to those without cirrhosis $(P<0.001)$. As expected, mortality was higher with higher CPT class, BCLC stage and use of curative therapies ( $P<0.05$ for all).

Figure 4 shows overall survival based on anti-viral therapy status. Survival was significantly higher in patients receiving anti-viral therapy (Figure 4A), and notably both among those with cirrhosis $(42 \%$ vs $25 \% ; P<0.001$; Figure $4 \mathrm{~B}$ ) and those without cirrhosis (58\% vs $36 \% ; P<0.001$; Figure $4 C$ ). Subgroup analysis of 5 -year survival based on anti-viral treatment status is shown in Table 3. Overall, patients receiving anti-viral therapy had greater five-year survival compared to untreated patients $(P<0.001)$. This trend was seen in patients with and without cirrhosis, as well as in all Child-Pugh classes among patients with cirrhosis. Patients receiving anti-viral therapy had higher survival than untreated patients with BCLC stages $0 / A, B$, and $C / D(P<0.001)$. These differences were also significant in patients receiving various cancer treatment types, from hepatic resection and liver transplant to tumour-directed treatment such as TACE/transarterial radioembolisation, and even among patients who received only supportive care $(P<0.05)$.

Patients receiving anti-viral therapy before HCC diagnosis may have improved access to medical care including HCC screening, which might result in lead time bias, so that the increased survival could merely reflect earlier diagnosis without improvement in outcomes. To address this question, we performed sensitivity analysis based on timing of anti-viral therapy, ie, only after HCC diagnosis vs before HCC diagnosis (Table S4). Patients receiving therapy before HCC diagnosis were older and had smaller maximum tumour size and more frequently had multifocal disease, vascular invasion, and extrahepatic metastases, as well as more advanced BCLC stage (Table S4). On analysis unadjusted for lead time, anti-viral therapy before HCC diagnosis was associated with decreased mortality vs anti-viral therapy only after HCC diagnosis (14.46 vs 19.85 deaths per 100 person-years, $P=0.0008$, Figure S2A and Table S5). This difference persisted after lead time analysis with estimated sojourn 70 and 140 days (Figure S2B-C), but not at a sojourn of 210 or 280 days (Figure S2D-E).

We also compared patients receiving anti-viral therapy only after HCC diagnosis with those not receiving anti-viral therapy at all. Here, there was no significant difference in maximum tumour size, proportion of multifocal tumours, or vascular invasion (Table S6). However, patients not receiving anti-viral therapy had greater proportion of extrahepatic metastasis and higher BCLC stage (42.2 vs 32.7\%; $P<0.05$ for both comparisons; Table S6). Screening is a related issue which may be related to access to care. Data on screening were available for 1224 patients (49\%). HCC screening rates in patients receiving no anti-viral therapy were lower than those in patients receiving anti-viral therapy before HCC diagnosis 
FIGURE 3 Indication for treatment with anti-viral therapy. For patients who were not on anti-viral therapy at time of $\mathrm{HCC}$ diagnosis, $\mathrm{y}$ axis shows percentage of patients for whom anti-viral therapy would have been indicated, based on local guidelines in the country to which the respective medical centres belong. Data are divided based on cirrhosis status: decompensated cirrhosis, compensated cirrhosis, and no cirrhosis. Local guidelines were defined as American Association for the Study of Liver Disease guidelines for United States centres, ${ }^{16}$ Ministry of Health and Welfare reimbursement criteria for Korean centres, ${ }^{27}$ and National Health Insurance Administration for the Taiwan centre $^{28}$

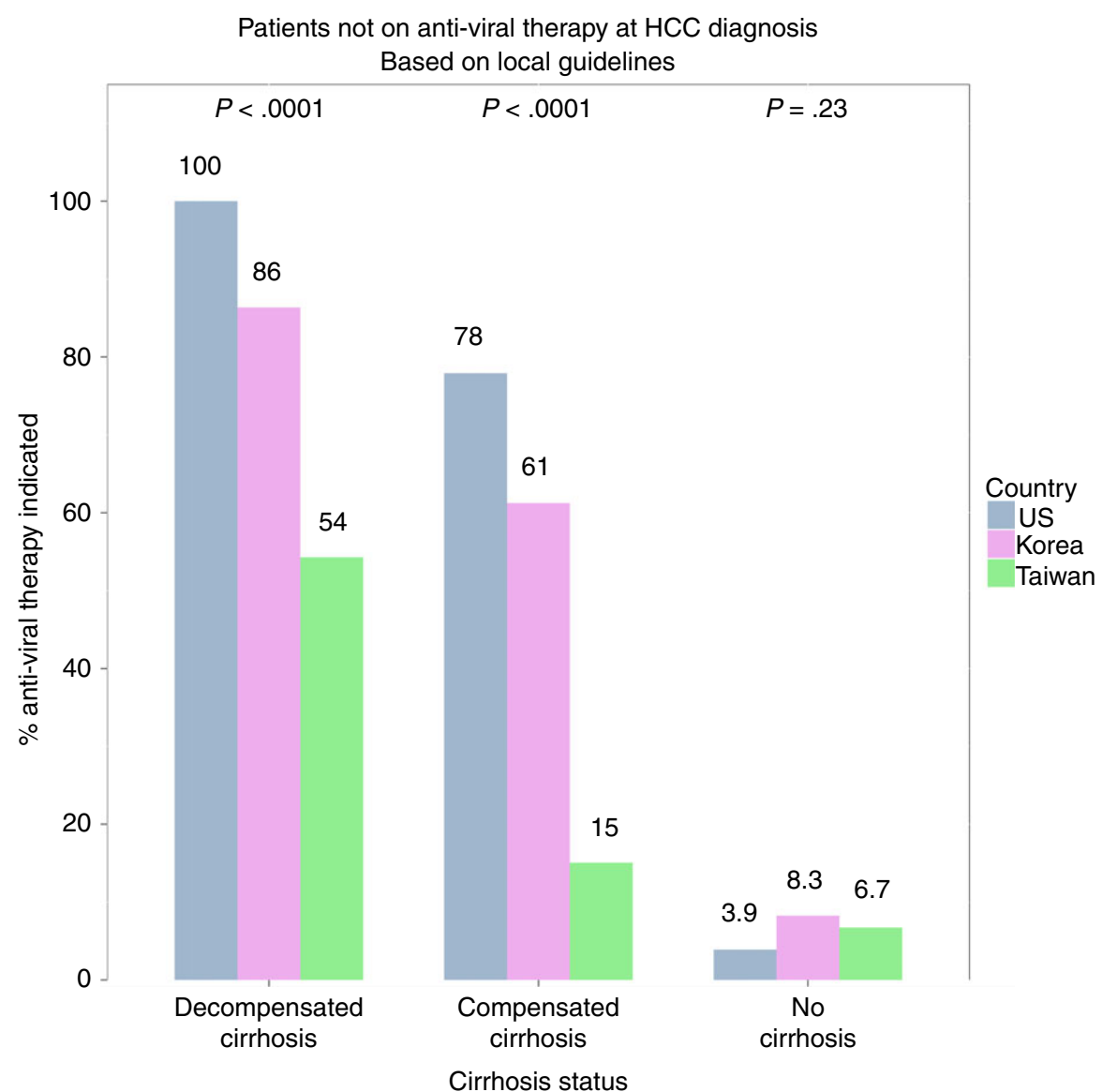

$(P<0.001)$ but were no different in patients receiving anti-viral therapy after HCC diagnosis ( $P=0.58)$.

\section{4 | Predictors of survival}

Table 4 shows predictors of mortality among HBV-related HCC patients. On unadjusted analysis, prognostic factors associated with increased mortality included younger age, male sex, cirrhosis, decompensated cirrhosis (CPT stage $B$ and $C$ ), higher MELD score, more advanced BCLC stage and the Taiwan centre ( $P<0.05$ for all). Conversely, factors associated with decreased morality included treatment with surgery (resection or liver transplant) or with either sorafenib or liver-directed therapy, anti-viral therapy at any time, duration of anti-viral therapy both before and after HCC diagnosis and anti-viral therapy with newer agents (entecavir or tenofovir) $(P<0.05$ for all). We included relevant predictors associated with mortality in the multiple regression models to estimate the adjusted $\mathrm{HR}$ and $95 \% \mathrm{Cl}$ of each predictor: age, sex, cirrhosis status, MELD, treatment type, BCLC stage and country. We also included anti-viral treatment status, stratified as no therapy, therapy only after HCC diagnosis and therapy before HCC diagnosis. In this model, anti-viral therapy either before or only after HCC diagnosis was independently associated with decreased mortality (adjusted HR 0.62 and 0.79 , respectively; $P<0.001$; Table 4). In this model, the Taiwan centre was no longer independently associated with increased mortality. On subanalysis of the patients for whom screening information was available, both screening and anti-viral therapy were associated with increased survival in a multivariate analysis model (Table S7).

\section{4 | DISCUSSION}

In this study, we characterised a cohort of patients with HBV-related HCC stratified by anti-viral therapy utilisation and cirrhosis status. We found that the use of anti-viral medications at any time in HBVrelated HCC patients was associated with a $20 \%-40 \%$ reduction in overall mortality of these patients, a sizable effect especially when compared to the modest survival benefits seen with many standard therapies for HCC such as palliative liver-directed therapy and sorafenib. ${ }^{31,32}$ The benefit of anti-viral therapy holds across a range of different cancer stages including BCLC stage $C / D$ and treatment types and even in patients receiving supportive care only. In addition, while there were significant differences in the rates of anti-viral utilisations and overall mortality among US vs Taiwan vs Korea centres, there was no difference in overall survival based on country of study sites in this multicentre international study after adjustment was made for anti-viral therapy use.

There is extensive evidence that anti-viral therapy in patients with $\mathrm{CHB}$ decreases risk of liver-related complications including liver decompensation and HCC development.,33,34 Our current study demonstrates that anti-viral therapy was associated with significantly reduced risk of death in a wide range of patients, from those 
TABLE 2 Overall mortality rates by various disease stage and treatment types

\begin{tabular}{|lcccc|}
\hline & Total & & $\begin{array}{l}\text { Person- } \\
\text { years of } \\
\text { fumber }\end{array}$ & $\begin{array}{l}\text { Mortality } \\
\text { (per 100 } \\
\text { person- }\end{array}$ \\
Group & 2518 & 1415 & 6384.24 & 22.2 \\
\hline Overall & & & & \\
\hline Cirrhosis & 681 & 312 & 1943.64 & 16.1 \\
\hline No cirrhosis & 1096 & 555 & 3187.25 & 17.4 \\
\hline Cirrhosis, Child-Pugh A & years)
\end{tabular}

TACE/TARE/XRT, Transarterial chemoembolisation/radioembolisation and external radiation therapy.

without cirrhosis to those with cirrhosis and advanced liver disease, from those with early to advanced cancer stage, and from those receiving curative therapy to those receiving only palliative therapy or even supportive care only. Anti-viral therapy could increase survival following HCC diagnosis in either the long- or short term through different mechanisms. In the long run, anti-viral therapy could decrease HCC recurrence and/or HCC progression. Previous studies showed anti-viral therapy was associated with decreased HCC recurrence and increased survival among patients with HBVrelated HCC undergoing surgery with curative intent.9,10 The longterm beneficial effects would be more significant in patients with early-stage HCC and compensated liver disease. In the short term, anti-viral therapy may counter the destabilising effect by HCC on liver function, which may be more important in patients with more advanced HCC and/or more impaired liver function. This study found that the increase in survival with anti-viral therapy was seen in a range of severity of liver disease and HCC stage, and, if anything, may have been more pronounced in patients with more advanced disease. In addition, choice of anti-viral therapy used may be important: use of newer anti-viral agents, ie, tenofovir or entecavir, was associated with improved survival compared to use of lamivudine or adefovir (Table 4).
(A)

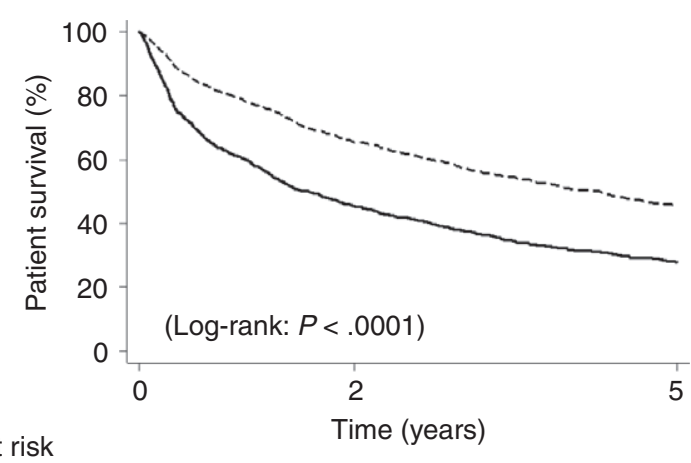

\begin{tabular}{rrrrrr} 
Number at risk & \multicolumn{5}{c}{ Time (years) } \\
No anti-virals & 1230 & $(608)$ & 427 & $(136)$ & 155 \\
Anti-virals & 1210 & $(391)$ & 656 & $(165)$ & 294
\end{tabular}

\section{- No antivirals _-.--. Antivirals}

(B)

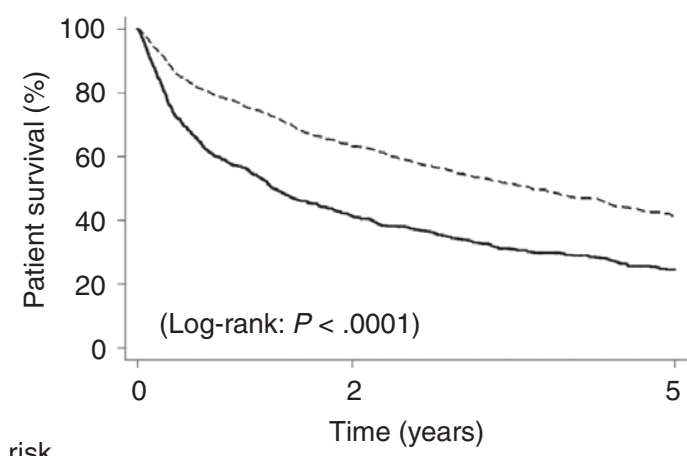

Number at risk

No anti-virals

\begin{tabular}{lllll}
873 & $(463)$ & 278 & $(92)$ & 101 \\
927 & $(323)$ & 493 & $(138)$ & 207 \\
\hline
\end{tabular}

(C)

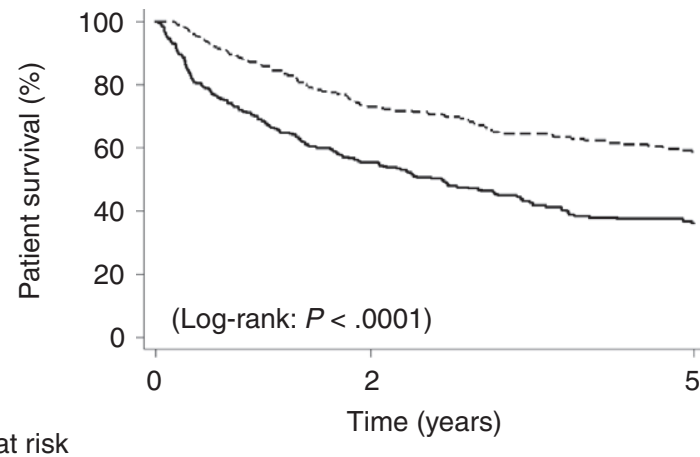

Number at risk

$\begin{array}{rccccc}\text { No anti-virals } & 357 & (145) & 149 & (44) & 54 \\ \text { Anti-virals } & 283 & (68) & 163 & (27) & 87 \\ & & & \end{array}$

FIGURE 4 Overall survival by anti-viral therapy. Overall survival for patients with hepatitis $B$ virus (HBV)-associated hepatocellular carcinoma (HCC), based on anti-viral therapy status. (A) Overall cohort. (B) Patients with cirrhosis. (C) Patients without cirrhosis. "No anti-virals" refers to patients who were never treated with anti-viral therapy directed at HBV, whereas "anti-virals" refers to treatment with anti-viral agents at any time

Disappointingly, in this multinational cohort, there was a strikingly low rate of anti-viral therapy. In particular, there was a much lower rate of anti-viral use in the Asian sites compared to the US 
TABLE 3 Five-year survival rates by disease stage and treatment types

\begin{tabular}{|llll} 
& \multicolumn{2}{c}{ Five-year survival (\%) } & \\
\cline { 2 - 3 } & $\begin{array}{l}\text { No anti-viral } \\
\text { therapy }\end{array}$ & $\begin{array}{c}\text { Anti-viral } \\
\text { therapy }\end{array}$ & P value \\
\hline Group & 27.9 & 45.3 & $<0.0001$ \\
\hline Cverall & & & \\
\hline Nirrhosis & 36.1 & 58.4 & $<0.0001$ \\
\hline Cirrhosis, Child-Pugh A & 34.8 & 50.5 & $<0.0001$ \\
\hline Cirrhosis, Child-Pugh B & 7.2 & 25.3 & $<0.0001$ \\
\hline Cirrhosis, Child-Pugh C & 7.2 & 29.8 & 0.0062 \\
\hline Barcelona clinic liver cancer stage & & \\
\hline O/A & 58.0 & 69.8 & 0.0002 \\
\hline B & 23.6 & 34.9 & 0.0003 \\
\hline C/D & 9.1 & 14.0 & $<0.0001$ \\
\hline Treatment & & & \\
\hline Resection & 64.9 & 74.5 & 0.0034 \\
\hline Liver transplant & 50.0 & 86.7 & 0.017 \\
\hline Ablative therapy & 67.2 & 63.3 & 0.89 \\
\hline TACE/TARE/XRT & 25.6 & 40.3 & $<0.0001$ \\
\hline Sorafenib & 9.3 & 9.4 & 0.44 \\
\hline Supportive care only & 7.1 & 11.3 & 0.037 \\
\hline
\end{tabular}

TACE/TARE/XRT, transarterial chemoembolisation/radioembolisation and external radiation therapy.

sites though anti-viral therapy was still severely underutilised in the US cohort with only $40 \%$ receiving anti-viral therapy at HCC diagnosis and only $68 \%$ total at any time. There are 2 potential explanations for these low uses: that patients did not meet local criteria for anti-viral therapy use (ie, existing guidelines did not recognise these patients as high-risk ${ }^{35,36}$ ) or that they did meet criteria but nonetheless did not receive anti-viral therapy. Our data suggest that both of these explanations may be true. Regarding the possibility of inadequacy of guidelines, we note that $<10 \%$ of patients without cirrhosis met any guideline criteria for anti-viral therapy despite developing HCC. Further, there is wide discrepancy between different guidelines in what proportion of patients with compensated cirrhosis would have met criteria for anti-viral therapy (Figures 2 and 3). These differences in guidelines on management of compensated cirrhosis with $\mathrm{CHB}$ one of the most prominent findings in this study suggest this may be a target for future guideline development.

Our data also suggest poor linkage-to-care. Among the patients not on treatment at time of HCC diagnosis, $>40 \%$ of those with cirrhosis met local and international criteria for anti-viral therapy (other than Taiwan reimbursement guidelines). This figure is even higher for decompensated cirrhosis. However, only $17 \%$ of patients were on anti-viral therapy at time of HCC diagnosis. This result is consistent with the poor linkage-to-care well known among HBV-infected patients with major gaps ranging from under screening and delayed diagnosis to suboptimal evaluation of patients with known HBV infection and undertreatment of patients who meet professional society guideline criteria for treatment. ${ }^{20-22}$ Inadequate linkage-to- care has other consequences as well: patients receiving anti-viral therapy before HCC diagnosis had higher rates of HCC screening and were diagnosed with $\mathrm{HCC}$ at an earlier stage, compared to among patients receiving anti-viral therapy only after HCC diagnosis or not at all. Further, on lead time bias analysis, an estimated sojourn in HCC diagnosis of at least 210 days (a highly conservative estimate) was needed to adjust for the difference in mortality between patients receiving anti-viral therapy before vs only after HCC diagnosis (Figure $\mathrm{S} 1$ ).

It should be noted that anti-viral treatment rate before $\mathrm{HCC}$ diagnosis was suboptimal in all of our study centres. This included US patients from 2 major university referral centres, which suggests that financial coverage is unlikely to be the only major barrier to anti-viral therapy in patients with chronic hepatitis B. In a prior study of more than 1000 mostly Asian American patients with CHB from the San Francisco Bay area (including Stanford University Medical Center), financial difficulty was the reason for no anti-viral therapy in under $10 \%$ of patients who met the American Association for the Study of Liver Diseases and/or US Panel guideline criteria for antiviral therapy. ${ }^{22}$ Rather, the most commonly cited reasons were the desire for further follow-up by patients and/or physicians and the perception that the patients' serum alanine aminotransferase levels were not elevated even though they met guideline criteria. ${ }^{22}$ Since $\mathrm{CHB}$ is a largely asymptomatic disease until onset of advanced HCC or end-stage liver disease, appropriate management often requires both patients and care providers to be better informed of the natural history of the disease and the need for regular monitoring and preventive therapy.

HBV-related HCC can occur in the absence of liver cirrhosis. According to prior studies, no overt cirrhosis is seen in $20 \%-40 \%$ of patients with HCC in primarily Asian cohorts ${ }^{37-40}$ and approximately $10 \%$ in non-Asian cohorts. ${ }^{41,42}$ In this study, we found that $27 \%$ of patients did not have recognisable cirrhosis. However, for those with cirrhosis, overall survival following HCC diagnosis was lower when compared to those without cirrhosis. This finding holds even when controlling for factors such as BCLC stage, treatment type and MELD score. The most likely explanation for this finding is that there is a higher incidence of second HCC development in patients with cirrhosis. Indeed, in this cohort, the 5-year survival in patients who underwent liver transplant was identical in patients with cirrhosis and those without cirrhosis ( $83 \%$ vs $86 \% ; P=0.91$ ), and the reason for this is likely that liver transplant is the only available treatment option which removed the diseased and precancerous livers. Very few transplants were performed in the Asian centres, which somewhat skews these data compared to what would be expected in a US cohort. It is important to note that patients with cirrhosis and anti-viral therapy demonstrated improved survival compared to their untreated counterparts, and anti-viral medications may be an important component of managing patients with cirrhosis and HCC.

One limitation of this study was that the vast majority of patients were of Asian ethnicity. Whether the findings can be applied to patients of other ethnicities requires further evaluation. Because of lack of longitudinal HBV DNA data, our data likely 
TABLE 4 Predictors of mortality of hepatocellular carcinoma patients with hepatitis B virus infection

\begin{tabular}{|c|c|c|c|c|}
\hline Characteristic & Unadjusted HR (95\% Cl) & $P$ value & Adjusted HR (95\% Cl) & $P$ value \\
\hline Age (per year) & $0.99(0.98-0.99)$ & 0.033 & $0.98(0.97-0.99)$ & $<0.001$ \\
\hline Male sex & $1.23(1.06-1.43)$ & 0.006 & $0.98(0.83-1.16)$ & 0.80 \\
\hline No cirrhosis & (Referent) & & (Referent) & \\
\hline Cirrhosis & $1.42(1.03-1.96)$ & 0.032 & $1.25(1.06-1.47)$ & 0.008 \\
\hline Cirrhosis, Child-Pugh C & $4.10(3.25-5.17)$ & $<0.001$ & & \\
\hline Model of end-stage liver disease score (per point) & $1.10(1.09-1.11)$ & $<0.001$ & $1.05(1.04-1.07)$ & $<0.001$ \\
\hline \multicolumn{5}{|l|}{ Treatment type } \\
\hline Supportive care only & (Referent) & & (Referent) & \\
\hline \multicolumn{5}{|l|}{ Diagnosis date } \\
\hline $2000-2005$ & (Referent) & & & \\
\hline $2006-2010$ & $1.07(0.93-1.23)$ & 0.35 & & \\
\hline 2011 and after & $0.89(0.76-1.03)$ & 0.12 & & \\
\hline \multicolumn{5}{|l|}{ Barcelona clinic liver cancer stage } \\
\hline O/A & (Referent) & & (Referent) & \\
\hline B & $2.94(2.49-3.46)$ & $<0.001$ & $2.47(2.04-2.99)$ & $<0.001$ \\
\hline$C / D$ & $8.41(7.23-9.77)$ & $<0.001$ & $5.86(4.91-7.00)$ & $<0.001$ \\
\hline \multicolumn{5}{|l|}{ Anti-viral therapy } \\
\hline \multicolumn{5}{|l|}{ Anti-viral type } \\
\hline Lamivudine or adefovir & (Referent) & & & \\
\hline Entecavir or tenofovir & $0.77(0.65-0.92)$ & 0.004 & & \\
\hline Other & $0.96(0.68-1.37)$ & 0.83 & & \\
\hline \multicolumn{5}{|l|}{ Country } \\
\hline United States & (Referent) & & (Referent) & \\
\hline Taiwan & $1.36(1.18-1.57)$ & $<0.001$ & $1.15(0.93-1.42)$ & 0.20 \\
\hline Korea & $0.85(0.72-1.02)$ & 0.054 & $0.99(0.78-1.26)$ & 0.93 \\
\hline
\end{tabular}

$\mathrm{HR}$, hazard ratio; $\mathrm{Cl}$, confidence interval; TACE/TARE/XRT, transarterial chemoembolisation/transarterial radioembolisation/external radiation therapy; RFA, radiofrequency ablation; PEA, percutaneous ethanol ablation.

underestimate the proportion of patients for whom therapy was ever indicated, since patients may have had higher HBV DNA concentration at an earlier date, which may argue against our claim that guidelines are inadequate for identifying high-risk patients. Finally, this study was retrospective in design, so we were not able to ascertain the reasons for lack of anti-viral therapy. A strength of this study was that it included a large number of HCC patients with HBV infection in Asian populations seen at both American as well as Asian centres. Further, all of the chart review procedures were standardised at each study site using the same case report form with similar definitions for the major outcome and predictor variables such as anti-viral therapy, liver cirrhosis and HCC.

In summary, we report here the largest cohort of diverse HBVrelated HCC patients from several medical centres from 3 countries. We found that anti-viral therapy at any time was significantly associated with $20 \%-40 \%$ lower mortality and this beneficial effect was independent of age, cirrhosis status, severity of cirrhosis, cancer stage and cancer treatment. Unfortunately, this study also found an 
alarmingly low rate of anti-viral therapy utilisation in centres in the US as well as Asia with the majority of patients not receiving any anti-viral therapy before their HCC diagnosis, even though a large proportion of them met both Asian and US treatment guideline criteria for therapy. Our data support more widespread use of anti-viral therapy in patients with HBV-related HCC, while highlighting the needs for improved linkage-to-care and earlier treatment with antiviral therapy in high-risk patients. In addition, the discrepancy between guidelines of management of patients with compensated cirrhosis has significant real-world implications on which patients are eligible for anti-viral therapy. Additional prospective studies are needed to understand and overcome the barriers to appropriate management of patients with HBV infection.

\section{ACKNOWLEDGEMENTS}

Declaration of personal interests: Vincent Chen, Ming-Lun Yeh, An Le, Mijung Jun, Waqar Saeed, Ju Dong Yang, Chung-Feng Huang, Hyo Young Lee, Pei-Chien Tsai, Nasra Giama, Jee Fu Huang, Nathan Kim, Pauline Nguyen, Hansen Dang, Hamdi Ali, Ning Zhang, Chia-Yen Dai, Lewis R. Roberts, Dae Won Jun: nothing to disclose. Wan-Long Chuang: Member of Advisory Board: Gilead, Abbvie, MSD, BMS, PharmaEssentia; Speaker: Gilead, Abbvie, MSD, BMS, PharmaEssentia. Young-Suk Lim: Grant/research support: Bayer Healthcare, Gilead Sciences. Speaker: Abbvie, Bayer Healthcare, BMS, Gilead Sciences, MSD. Advisory board or consultation: Abbvie, Bayer Healthcare, BMS, Gilead Sciences, MSD. Ming-Lung Yu: Grant/research support: Abbvie, Abbott, Bristol-Myers Squibb, Gilead, Merck, Roche. Speakers' bureau: Abbvie, Abbott, Ascletis, Bristol-Myers Squibb, Gilead, Merck, Pharmaessential, Roche. Advisory board or consultation: Abbvie, Abbott, Ascletis, Bristol-Myers Squibb, Gilead, J\&J, Merck, Novartis, Pharmaessential, Roche. Mindie Nguyen: Research support: Bristol-Myers Squibb, Gilead, Pfizer, Janssen, Novartis, B.K. Kee Foundation, Asian Health Foundation, National Cancer Institute. Consultant and/or an advisory board: Novartis, Anylam, Dynavax, Bayer, Intercept, Roche, Gilead, BristolMyers Squibb, and Janssen. Writing assistance, None.

Declaration of funding interests: MHL was supported in part by grant MOST 105-2628-B-010-003 MY4.

\section{AUTHORSHIP}

Guarantor of the article: Mindie H. Nguyen.

Author's contributions: Vincent Chen: study design, data collection, data analysis and interpretation, and drafting of the manuscript; Ming-Lun Yeh: data collection, data interpretation, and critical review of the manuscript; An Le: data analysis and interpretation, and critical review of the manuscript. Mijung Jun: data collection, data interpretation, and critical review of the manuscript. Waqar Saeed: data collection, data interpretation, and critical review of the manuscript. Ju Dong Yang: data collection and interpretation, and critical review of the manuscript. Chung-Feng Huang: data collection and interpretation, and critical review of the manuscript. Hyo Young Lee: data collection, data interpretation, and critical review of the manuscript. Pei-Chien Tsai: data collection, data interpretation, and critical review of the manuscript. Mei-Hsuan Lee: data analysis and interpretation and critical revision of the manuscript. Nasra Giama: data collection and interpretation, and critical review of the manuscript. Nathan Kim: data collection, data interpretation, and critical review of the manuscript. Jee-Fu Huang: data collection and interpretation, and critical review of the manuscript. Chia-Yen Dai: data collection and interpretation, and critical review of the manuscript. Wan-Long Chuang: data collection and interpretation, and critical review of the manuscript. Lewis Roberts: data collection and interpretation, and critical review of the manuscript. Dae Won Jun: data collection and interpretation, and critical review of the manuscript. Young-Suk Lim: study design, data collection and interpretation, and critical review of the manuscript. Ming-Lung Yu: study design, data collection and interpretation, and critical review of the manuscript. Mindie Nguyen: concept development, study design, data collection, data analysis and interpretation, and critical revision of the manuscript. All authors identified above have critically reviewed the paper and approve the final version of this paper, including the authorship statement.

\section{ORCID}

V. L. Chen iD http://orcid.org/0000-0002-0157-6066

C.-F. Huang iD http://orcid.org/0000-0002-3367-068X

D. W. Jun (iD http://orcid.org/0000-0002-2875-6139

M.-L. Yu iD http://orcid.org/0000-0001-8145-1900

M. H. Nguyen (iD http://orcid.org/0000-0002-6275-4989

\section{REFERENCES}

1. American Cancer Society. Global Cancer Facts \& Figures. Atlanta: American Cancer Society; 2011.

2. Beasley RP, Lin C-C, Hwang L-Y, Chien C-S. Hepatocellular carcinoma and hepatitis B virus. A prospective study of 22707 men in Taiwan. Lancet. 1981:2:1129-1133.

3. Poon RT, Fan ST, Lo CM, Liu CL, Ng IO, Wong J. Long-term prognosis after resection of hepatocellular carcinoma associated with hepatitis B-related cirrhosis. J Clin Oncol. 2000;18:1094-1101.

4. Kim JM, Kwon CH, Joh JW, et al. Differences between hepatocellular carcinoma and hepatitis B virus infection in patients with and without cirrhosis. Ann Surg Oncol. 2014;21:458-465.

5. Lai CL, Yuen MF. Prevention of hepatitis B virus-related hepatocellular carcinoma with antiviral therapy. Hepatology. 2013;57:399-408.

6. Liaw Y-F, Sung JJY, Chow WC, et al. Lamivudine for patients with chronic hepatitis B and advanced liver disease. $N$ Engl J Med. 2004;351:1521-1531.

7. Coffin CS, Rezaeeaval M, Pang JX, et al. The incidence of hepatocellular carcinoma is reduced in patients with chronic hepatitis B on long-term nucleos(t)ide analogue therapy. Aliment Pharmacol Ther. 2014;40:1262-1269.

8. Chong CCN, Wong GLH, Wong VWS, et al. Antiviral therapy improves post-hepatectomy survival in patients with hepatitis B virus-related hepatocellular carcinoma: a prospective-retrospective study. Aliment Pharmacol Ther. 2015;41:199-208. 
9. Yin J, Li N, Han Y, et al. Effect of antiviral treatment with nucleotide/nucleoside analogs on postoperative prognosis of hepatitis $\mathrm{B}$ virus-related hepatocellular carcinoma: a two-stage longitudinal clinical study. J Clin Oncol. 2013;31:3647-3655.

10. Wong JS, Wong GL, Tsoi KK, et al. Meta-analysis: the efficacy of anti-viral therapy in prevention of recurrence after curative treatment of chronic hepatitis B-related hepatocellular carcinoma. Aliment Pharmacol Ther. 2011;33:1104-1112.

11. Wu C-Y, Chen Y-J, Ho HJ, et al. Association between nucleoside analogues and risk of hepatitis B virus-related hepatocellular carcinoma recurrence following liver resection. J Amer Med Assoc. 2012;308:1906.

12. Lee TY, Lin JT, Zeng YS, Chen YJ, Wu MS, Wu CY. Association between nucleos(t)ide analog and tumor recurrence in hepatitis $B$ virus-related hepatocellular carcinoma after radiofrequency ablation. Hepatology. 2016;63:1517-1527.

13. $\mathrm{Xu} X$, Huang $\mathrm{P}$, Tian $\mathrm{H}$, et al. Role of lamivudine with transarterial chemoembolization in the survival of patients with hepatocellular carcinoma. J Gastroenterol Hepatol. 2014;29:1273-1278.

14. Xu L, Gao H, Huang J, et al. Antiviral therapy in the improvement of survival of patients with hepatitis B virus-related hepatocellular carcinoma treated with sorafenib. J Gastroenterol Hepatol. 2015;30: 1032-1039.

15. Yang $\mathrm{Y}, \mathrm{Wen} \mathrm{F}$, Li J, et al. A high baseline HBV load and antiviral therapy affect the survival of patients with advanced HBV-related HCC treated with sorafenib. Liver Int. 2015;35:2147-2154.

16. Terrault NA, Bzowej NH, Chang KM, et al. AASLD guidelines for treatment of chronic hepatitis B. Hepatology. 2016;63:261-283.

17. Liaw $\mathrm{YF}, \mathrm{Kao} \mathrm{JH}$, Piratvisuth $\mathrm{T}$, et al. Asian-Pacific consensus statement on the management of chronic hepatitis B: a 2012 update. Hepatol Int. 2012;6:531-561.

18. European Association for the Study of the Liver. EASL 2017 Clinical Practice Guidelines on the management of hepatitis B virus infection. J Hepatol. 2017;67:370-398.

19. Lim SG, Amarapurkar DN, Chan HL, et al. Reimbursement policies in the Asia-Pacific for chronic hepatitis B. Hepatol Int. 2015;9:43-51.

20. Liou IW, Nguyen MH. The cascade of care in chronic hepatitis B. Curr Hepatol Rep. 2016;15:209-219.

21. Ku KC, Li J, Ha NB, Martin M, Nguyen VG, Nguyen MH. Chronic hepatitis $\mathrm{B}$ management based on standard guidelines in community primary care and specialty clinics. Dig Dis Sci. 2013;58:3626-3633.

22. Kim LH, Nguyen VG, Trinh HN, Li J, Zhang JQ, Nguyen MH. Low treatment rates in patients meeting guideline criteria in diverse practice settings. Dig Dis Sci. 2014;59:2091-2099.

23. Bruix J, Sherman M. Management of hepatocellular carcinoma: an update. Hepatology. 2011;53:1020-1022.

24. Park JW, Chen M, Colombo M, et al. Global patterns of hepatocellular carcinoma management from diagnosis to death: the BRIDGE Study. Liver Int. 2015;35:2155-2166.

25. National Center for Health Statistics. National Death Index. https:// www.cdc.gov/nchs/ndi/index.htm. Accessed March 26, 2018.

26. Sarin SK, Kumar M, Lau GK, et al. Asian-Pacific clinical practice guidelines on the management of hepatitis B: a 2015 update. Hepatol Int. 2016;10:1-98.

27. Ministry of Health and Welfare. Details of the application criteria and methods of medical treatment benefits. http://www.mohw.go.

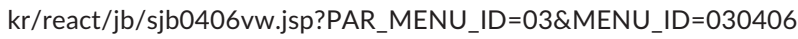
\&CONT_SEQ=342581\&page=1. Accessed March 26, 2018

28. National Health Insurance Administration. Drug reimbursement regulations. https://www.nhi.gov.tw/Content_List.aspx?n=E70D4F1 BD029DC37\&topn=3FC7D09599D25979. Accessed March 26, 2018

29. Duffy SW, Nagtegaal ID, Wallis M, et al. Correcting for lead time and length bias in estimating the effect of screen detection on cancer survival. Am Journal Epidemiol. 2008;168:98-104.
30. Thein H-H, Campitelli MA, Yeung LT, Zaheen A, Yoshida EM, Earle CC. Improved survival in patients with viral hepatitis-induced hepatocellular carcinoma undergoing recommended abdominal ultrasound surveillance in Ontario: a population-based retrospective cohort study. PLoS ONE. 2015;10:e0138907.

31. Llovet JM, Real MI, Montaña X, et al. Arterial embolisation or chemoembolisation versus symptomatic treatment in patients with unresectable hepatocellular carcinoma: a randomised controlled trial. The Lancet. 2002;359:1734-1739.

32. Llovet JM, Ricci S, Mazzaferro V, et al. Sorafenib in advanced hepatocellular carcinoma. N Engl J Med. 2008;359:378-390.

33. Lin D, Yang HI, Nguyen N, et al. Reduction of chronic hepatitis Brelated hepatocellular carcinoma with anti-viral therapy, including low risk patients. Aliment Pharmacol Ther. 2016;44:846-855.

34. Lok AS, McMahon BJ, Brown RS Jr, et al. Antiviral therapy for chronic hepatitis B viral infection in adults: a systematic review and meta-analysis. Hepatology. 2016;63:284-306.

35. Tong MJ, Hsien C, Hsu L, Sun HE, Blatt LM. Treatment recommendations for chronic hepatitis $\mathrm{B}$ : an evaluation of current guidelines based on a natural history study in the United States. Hepatology. 2008;48:1070-1078.

36. Chen TM, Chang CC, Huang PT, Wen CF, Lin CC. Performance of risk estimation for hepatocellular carcinoma in chronic hepatitis $\mathrm{B}$ (REACHB) score in classifying treatment eligibility under 2012 Asian Pacific Association for the Study of the Liver (APASL) guideline for chronic hepatitis B patients. Aliment Pharmacol Ther. 2013;37:243-251.

37. Kumar M, Kumar R, Hissar SS, et al. Risk factors analysis for hepatocellular carcinoma in patients with and without cirrhosis: a case-control study of 213 hepatocellular carcinoma patients from India. J Gastroenterol Hepatol. 2007;22:1104-1111.

38. Kwak HW, Park JW, Nam BH, et al. Clinical outcomes of a cohort series of patients with hepatocellular carcinoma in a hepatitis B virus-endemic area. J Gastroenterol Hepatol. 2014;29:820-829.

39. Chen $\mathrm{CH}$, Su WW, Yang SS, et al. Long-term trends and geographic variations in the survival of patients with hepatocellular carcinoma: analysis of 11,312 patients in Taiwan. J Gastroenterol Hepatol. 2006;21:1561-1566.

40. Chen VL, Le AK, Kim NG, et al. Effects of cirrhosis on shortterm and long-term survival of patients with hepatitis B-related hepatocellular carcinoma. Clin Gastroenterol Hepatol. 2016;14:887-895. e1.

41. Yang JD, Kim WR, Coelho R, et al. Cirrhosis is present in most patients with hepatitis B and hepatocellular carcinoma. Clin Gastroenterol Hepatol. 2011;9:64-70.

42. Chayanupatkul M, Omino R, Mittal S, et al. Hepatocellular carcinoma in the absence of cirrhosis in patients with chronic hepatitis $B$ virus infection. J Hepatol. 2017;66:355-362.

\section{SUPPORTING INFORMATION}

Additional supporting information will be found online in the Supporting Information section at the end of the article.

How to cite this article: Chen VL, Yeh M-L, Le AK, et al. Anti-viral therapy is associated with improved survival but is underutilised in patients with hepatitis B virus-related hepatocellular carcinoma: real-world east and west experience. Aliment Pharmacol Ther. 2018;48:44-54. https://doi.org/10.1111/apt.14801 